\title{
Whole-transcriptome sequencing reveals heightened inflammation and defective host defence responses in chronic rhinosinusitis with nasal polyps
}

\author{
Yang Peng ${ }^{1,2,3,8}$, Xiao-Xue Zi $i^{1,4,8}$, Teng-Fei Tian ${ }^{1,5,8}$, Bernett Lee ${ }^{6}$, \\ Josephine Lum ${ }^{6}$, See Aik Tang ${ }^{6}$, Kai Sen Tan ${ }^{1}$, Qian-Hui Qiu ${ }^{2,7}$, Jing Ye $^{5}$, Li Shi ${ }^{4}$, \\ Wei-Jie Guan ${ }^{1,3}$, Anand Kumar Andiappan ${ }^{6}$ and De Yun Wang (i) ${ }^{1}$
}

@ERSpublications

Whole-transcriptome sequencing shows gene signatures associated with interferon signalling response and host defence are implicated in chronic rhinosinusitis with nasal polyps, opening up new opportunities for therapeutic interventions http://bit.ly/2KyF3dT

Cite this article as: Peng $\mathrm{Y}, \mathrm{Zi} \mathrm{X}-\mathrm{X}$, Tian T-F, et al. Whole-transcriptome sequencing reveals heightened inflammation and defective host defence responses in chronic rhinosinusitis with nasal polyps. Eur Respir J 2019; 54: 1900732 [https://doi.org/10.1183/13993003.00732-2019].

\begin{abstract}
Introduction: The pathways underlying chronic rhinosinusitis with nasal polyps (CRSwNP) are unclear. We conducted genome-wide gene expression analysis to determine pathways and candidate gene sets associated with CRSwNP.

Methods: We performed whole-transcriptome RNA sequencing on 42 polyp (CRSwNP-NP) and 33 paired nonpolyp inferior turbinate (CRSwNP-IT) tissues from patients with CRSwNP and 28 inferior turbinate samples from non-CRS controls (CS-IT). We analysed the differentially expressed genes (DEGs) and the gene sets that were enriched in functional pathways.

Results: Principal component-informed analysis revealed cilium function and immune regulation as the two main Gene Ontology (GO) categories differentiating CRSwNP patients from controls. We detected 6182 and 1592 DEGs between CRSwNP-NP versus CS-IT and between CRSwNP-NP versus CRSwNP-IT tissues, respectively. Atopy status did not have a major impact on gene expression in various tissues. GO analysis on these DEGs implicated extracellular matrix (ECM) disassembly, O-glycan processing, angiogenesis and host viral response in CRSwNP pathogenesis. Ingenuity Pathway Analysis identified significant enrichment of type 1 interferon signalling and axonal guidance canonical pathways, angiogenesis, and collagen and fibrotic changes in CRSwNP (CRSwNP-NP and CRSwNP-IT) tissues compared with CS-IT. Finally, gene set enrichment analysis implicated sets of genes co-regulated in processes associated with inflammatory response and aberrant cell differentiation in polyp formation.

Conclusions: Gene signatures involved in defective host defences (including cilia dysfunction and immune dysregulation), inflammation and abnormal metabolism of ECM are implicated in CRSwNP. Functional validation of these gene expression patterns will open opportunities for CRSwNP therapeutic interventions such as biologics and immunomodulators.
\end{abstract}




\section{Introduction}

Chronic rhinosinusitis (CRS) is a debilitating inflammatory disease of the nasal mucosa [1]. Nasal polyps (NP) are the most common comorbidity, affecting 30\% of patients with CRS [2], and cause a considerable socioeconomic burden and impaired quality of life [3]. Clinical management of CRS with NP (CRSwNP) is largely ineffective, partly due to the limited understanding of the underlying pathogenic factors and a paucity of effective therapeutic interventions $[4,5]$. Understanding the mechanisms that underlie CRSwNP pathogenesis may help identify targets in critical candidate pathways for therapeutic interventions.

The known biological processes implicated in CRSwNP are multifaceted, including airway inflammation [2], tight junction impairment [6], pathogen infection [7, 8] and a defective host defence [9-13]. The complexity of gene expression patterns in CRSwNP has not been adequately addressed, partly because the reference tissues often used have been suboptimal for biomarker profiling. For instance, differences in gene signatures between NP and control tissues, i.e. inferior turbinate (IT), from healthy subjects might stem from genetic or environmental factors, or both [14-17]. Additionally, Gene Ontology (GO) analyses have mostly been based on differentially expressed genes (DEGs) identified by microarray assays, but not on gene sets or pathways [18]. Finally, the limited sample sizes have markedly decreased the statistical power for gene signature profiling $[14,15]$. ORdovas-Montanes et al. [19], however, reported strong type 2 immune-mediated signatures associated with barrier tissue dysfunction in NP, despite a relatively small sample size that did not compromise the analysis because of deep single-cell profiling. Whole-genome approaches that use well-powered sample sizes are necessary to thoroughly elucidate gene signatures in CRSwNP.

RNA sequencing is a powerful means to explore candidate transcripts in an unbiased manner [20-22], and to identify cellular and molecular pathways. We hypothesised that the gene signatures and pathways identified by RNA sequencing would differ considerably between pairwise comparisons of the IT of patients with CRSwNP and control subjects. In this study, we thus performed RNA sequencing to identify the transcriptomic signatures associated with CRSwNP, by incorporating a three-way comparison of the nasal tissues from CRSwNP patients and controls as well as a comprehensive analytical pipeline to determine the DEGs and gene sets that enriched in functional pathways. The gene signatures and candidate pathways would help identify disease targets (i.e. abnormal matrix metabolism, immune dysfunction) and novel treatment options such as biologics for CRSwNP [14-16, 18, 20].

\section{Methods}

\section{Study design and participants}

Subjects were recruited from individual sites and provided written informed consent. The Institutional Review Boards of Guangdong General Hospital (Guangzhou, China), The Second Hospital of Shandong University (Jinan, China), The First Affiliated Hospital of Nanchang University (Nanchang, China) and the National Healthcare Group Domain Specific Review Board of Singapore (Singapore) approved the study protocol.

CRSwNP diagnosis was based on the European Position Paper on Rhinosinusitis and Nasal Polyps 2012 [1]. All CRSwNP patients had bilateral NP (scored 1-4 points bilaterally based on the NP size score, evaluated via nasal endoscopy) [23, 24]. Patients with antrochoanal polyps, fungal sinusitis and/or recurrent lower airway infections were excluded. None of the participants had CRS without NP or aspirin-exacerbated respiratory diseases. We recruited control subjects who underwent septoplasty for anatomical variations without otolaryngologist-diagnosed CRS (free from clinical symptoms; confirmed via inspection of nasal endoscopy). Neither atopy nor allergic rhinitis were mandatory for the exclusion criteria for both CRSwNP

Affiliations: ${ }^{1}$ Dept of Otolaryngology, Yong Loo Lin School of Medicine, National University of Singapore, Singapore. ${ }^{2}$ Dept of Otolaryngology, Zhujiang Hospital, Southern Medical University, Guangzhou, Guangdong, China. ${ }^{3}$ State Key Laboratory of Respiratory Disease, National Clinical Research Center for Respiratory Disease, Guangzhou Institute of Respiratory Health, The First Affiliated Hospital of Guangzhou Medical University, Guangzhou Medical University, Guangzhou, Guangdong, China. ${ }^{4}$ Shandong Provincial ENT Hospital Affiliated to Shandong University, Shandong University, Jinan, China. ${ }^{5}$ Dept of Otolaryngology, The First Affiliated Hospital of Nanchang University, Nanchang, Jiangxi, China. ${ }^{6}$ Singapore Immunology Network (SIgN), Agency for Science, Technology and Research (A*STAR), Singapore. ${ }^{7}$ Dept of Otolaryngology Head and Neck Surgery, Guangdong General Hospital, Guangdong Academy of Medical Sciences, Guangzhou, China. ${ }^{8}$ These authors contributed equally to this work.

Correspondence: Anand Kumar Andiappan, Singapore Immunology Network (SIgN), Agency for Science, Technology and Research (A*STAR), \#04-06, 8A Biomedical Grove, 138648 Singapore. E-mail: anand_andiappandimmunol.a-star.edu.sg. Wei-Jie Guan, State Key Laboratory of Respiratory Disease, National Clinical Research Center for Respiratory Disease, Guangzhou Institute of Respiratory Health, The First Affiliated Hospital of Guangzhou Medical University, Guangzhou Medical University, 151 Yanjiang Road, Guangzhou, Guangdong, China. E-mail: battery203a163.com 
patients and control subjects. Furthermore, none of the study participants had an upper respiratory tract infection or had used intranasal or systemic corticosteroids or antibiotics within 4 weeks before the surgery. In total, we recruited $44 \mathrm{CRSwNP}$ patients and 41 control subjects. All but three study participants (Singaporeans) were of Chinese ethnicity. Data from 42 CRSwNP-NP samples, 33 CRSwNP-IT samples and 28 CS-IT samples passed the initial quality control of RNA sequencing and were therefore included in further analysis.

In this cross-sectional study (figure 1), paired IT and NP was biopsied from patients with CRSwNP, and IT was biopsied from control subjects. Following biopsy, tissues were immediately split for RNA sequencing and histological assessment. Haematoxylin/eosin staining and immunohistochemistry were performed as previously described for eosinophil and neutrophil enumeration of paraffin-embedded slides, respectively [25]. Eosinophilic or neutrophilic NP denoted the percentage of eosinophils or neutrophils $>10 \%$, respectively $[25,26]$.

\section{RNA sequencing and data processing}

Tissues were pre-treated with RNAlater (Thermo Fisher Scientific, Waltham, MA, USA) for overnight incubation at $4^{\circ} \mathrm{C}$ and then stored in $-80^{\circ} \mathrm{C}$ freezers before RNA extraction. Total RNA was extracted by a double extraction protocol: RNA isolation by acid guanidinium thiocyanate-phenol-chloroform extraction (TRIzol; Thermo Fisher Scientific), followed by RNeasy Micro clean-up (Qiagen, Hilden, Germany). All human RNAs were analysed on an Agilent Bioanalyzer (Agilent, Santa Clara, CA, USA) for quality assessment by RNA integrity number (range 5.8-9.1; median 7.3). cDNA libraries were prepared with $2 \mathrm{ng}$ total RNA input and $1 \mu \mathrm{L}$ of a 1:50000 dilution of ERCC RNA Spike-in Controls (Thermo Fisher Scientific) using the SMARTSeq version 2 protocol with the following modifications: 1) $20 \mu \mathrm{M}$ template switch oligonucleotides and 2) 250 pg cDNA with 1/5 reaction of a Nextera XT kit (Illumina, San Diego, CA, USA). The length distribution of the cDNA libraries was monitored using a DNA High Sensitivity Reagent Kit on a Labchip GX system (Perkin Elmer, Waltham, MA, USA). All samples were subjected to an indexed paired-end sequencing run of $2 \times 151$ cycles on an HiSeq 4000 platform (Illumina) (30 samples per lane).

\section{Statistical analyses}

We expected the gene signatures identified through whole-transcriptome sequencing to be independent of the CRSwNP phenotypes. To eliminate interindividual differences arising from genetic or environmental

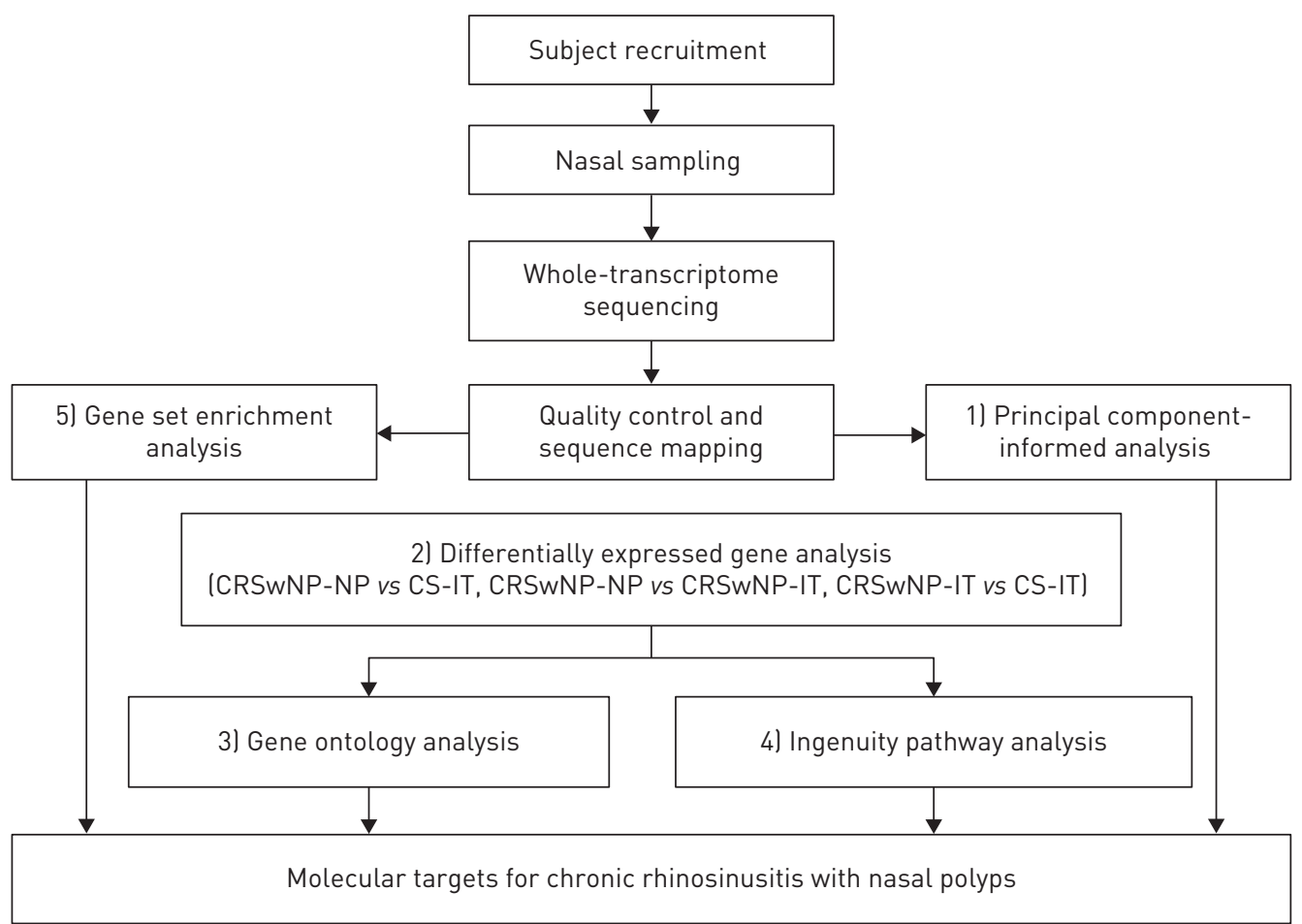

FIGURE 1 Study workflow from subject recruitment and sample collection to data analysis and interpretation. CRSwNP-NP: nasal polyps tissues from patients with chronic rhinosinusitis with nasal polyps; CS-IT: inferior turbinate in control subjects; CRSwNP-IT: paired inferior turbinate tissues from patients with chronic rhinosinusitis with nasal polyps. More information on the detailed methods is provided in the Methods section. 
factors, we conducted a three-way comparison between the IT in control subjects (CS-IT) versus the paired NP (CRSwNP-NP) and IT (CRSwNP-IT) in patients with CRSwNP. To minimise the heterogeneity of the study population, we recruited participants without physician-diagnosed or self-reported asthma. We stratified patients with CRSwNP by different inflammatory phenotypes (i.e. eosinophilic or neutrophilic NP phenotypes) to determine whether the inflammatory phenotypes markedly confound gene expression patterns. We recruited an adequate sample size of participants ( $\mathrm{n}>25$ for each group) to ensure sufficient statistical power to investigate the interactions of different gene sets and pathways. Importantly, we designed an analytical pipeline to identify different gene signatures between CRSwNP-IT and CS-IT within the same individuals with CRSwNP. These novel study designs have collectively underpinned the validity of our findings.

HiSeq FASTQ files were mapped to the human genome build hg38 using STAR (splice junction annotations based on GENCODE version 26; www.gencodegenes.org). Gene counts were obtained with featureCounts using GENCODE version 26 annotations. Principal component analysis (PCA) was carried out in $\mathrm{R}$ version 3.3 .3 (www.r-project.org) using $\log _{2}$ reads per kilobase million (RPKM) to determine the differences between the samples in each group. DEG analysis was performed using edgeR as pairwise comparisons on genes filtered for $\log _{2}$ RPKM interquartile range $>0.5$. We presented the adjusted p-values to derive more conservative significance thresholds given the large number of tests performed. We corrected for multiple testing with the Benjamini-Hochberg algorithm (adjusted p-value $<0.05$ was considered statistically significant). Venn diagrams were generated using $\mathrm{R}$ software. GO enrichment analysis using the Bioconductor (www.bioconductor.org) package TopGO was performed to identify biological processes and protein functional groups enriched in groups of DEGs (background was the filtered list of genes used for DEG analysis). Ingenuity Pathway Analysis (www.nihlibrary.nih.gov/ resources/tools/ingenuity-pathways-analysis-ipa) was used to define gene sets-determined pathways associated with DEGs. Significance threshold was set to default. Gene Set Enrichment Analysis (GSEA) was conducted using the Bioconductor package fgsea to identify gene sets of enrichment patterns using the Molecular Signatures Database (MSigDB; http://software.broadinstitute.org/gsea/msigdb). All software was run using default parameters, unless otherwise stated.

\section{Results}

Subject recruitment and clinical characteristics

We recruited $44 \mathrm{CRSwNP}$ patients and 41 control subjects whose surgically resected samples were subject to RNA sequencing. Overall, CRSwNP patients were older than control subjects and had a lower proportion of atopy (both $\mathrm{p}<0.05$ ) (supplementary table E1). 15 patients with CRSwNP $(34.1 \%)$ had tissue eosinophilia. The mean \pm SD NP size score was $4.7 \pm 1.1$. None of the study participants had physician-diagnosed or self-reported asthma. Subanalysis of the 42 CRSwNP patients and 28 control subjects whose RNA sequencing data passed the initial quality control is also shown in supplementary table E1, which was not materially altered compared with that of the overall study population.

\section{Global transcriptomic profiling reveals that ciliary function and immunomodulation segregate the nasal tissues}

We first evaluated the overall distribution of gene expression across the three tissues types by PCA on the 103 tissue samples that passed quality control standards (figure 2). No technical effects in PCA were identified. Confounders such as batch effects, RNA integrity number and sample collection processes did not confer any major influence on the analysis. However, the global expression patterns revealed a spatial separation on the PCA matrix based on tissue category, with the tissues from the CRSwNP patients separating from the control subject tissues. The strongest variance was observed between CRSwNP-NP and CS-IT, and a comparatively minor separation was observed between the nonpolyp CRSwNP-IT and CS-IT tissues (figure 2a-c).

We then analysed the top loading genes accounting for this observed variance (supplementary table E2). The first principal component (PC1) comprised genes regulating ciliogenesis and ciliary function (i.e. cilium assembly, ultrastructural protein assembly, cilium motility, intraciliary transport), whereas PC2 mainly consisted of genes modulating inflammatory and immune responses (i.e. chemokine-mediated signalling, innate and adaptive immune response, T-cell proliferation, co-stimulation), and collagen and extracellular matrix (ECM) metabolism (i.e. complex of collagen trimmers, ECM structural constituent and organisation) (figure $2 \mathrm{~d}$ and e). We then performed a GO analysis to further evaluate these identified genes using the known evidence from previously reported associations.

Next, we performed clustering analysis on the global gene expression data to further examine the heterogeneity of gene expression among individual participants independent of their disease status. Here, we observed tighter clustering between CRSwNP-NP and CRSwNP-IT subjects than with CS-IT 
a)

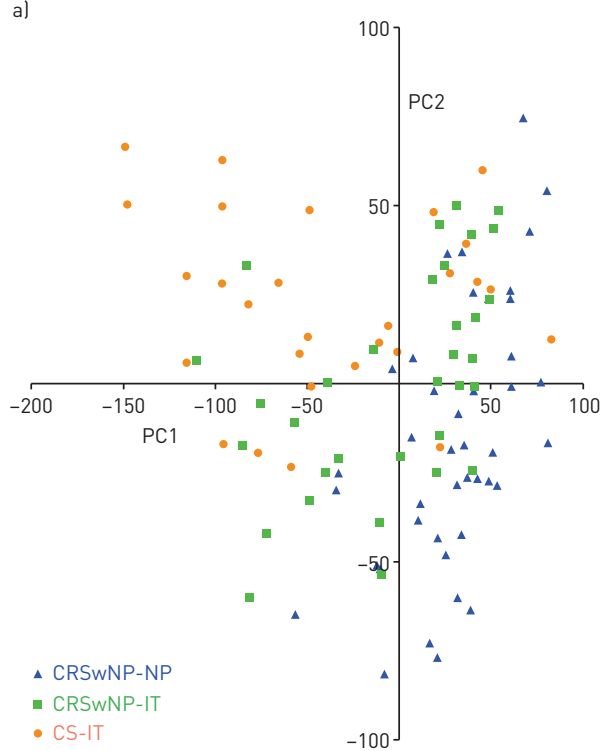

b)

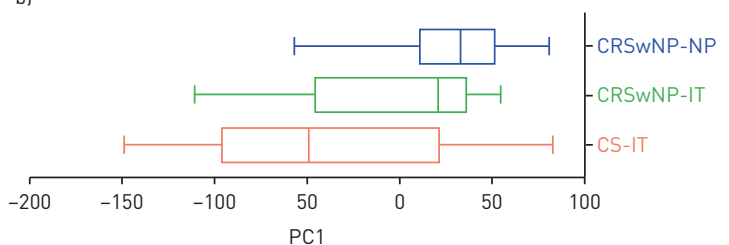

d)

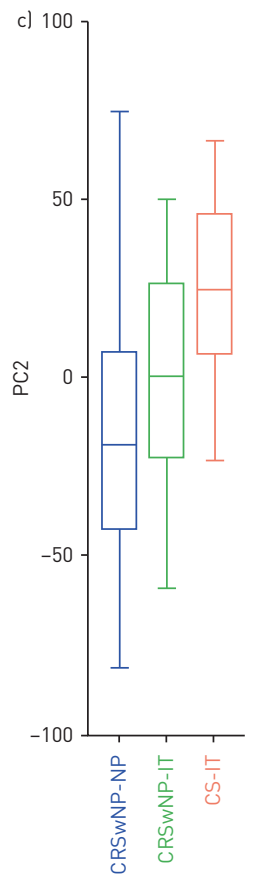

Cilium-dependent cell motility

PLC-activating GPCR signalling pathway

Intraciliary transport

O-glycan processing

Epithelial cell differentiation Negative regulation of endopeptidase activity

Outer dynein arm assembly

Axoneme assembly

Determination of left/right symmetry

Motile cilium assembly

Inner dynein arm assembly

Epithelial cilium movement

Cilium movement

Cilium assembly

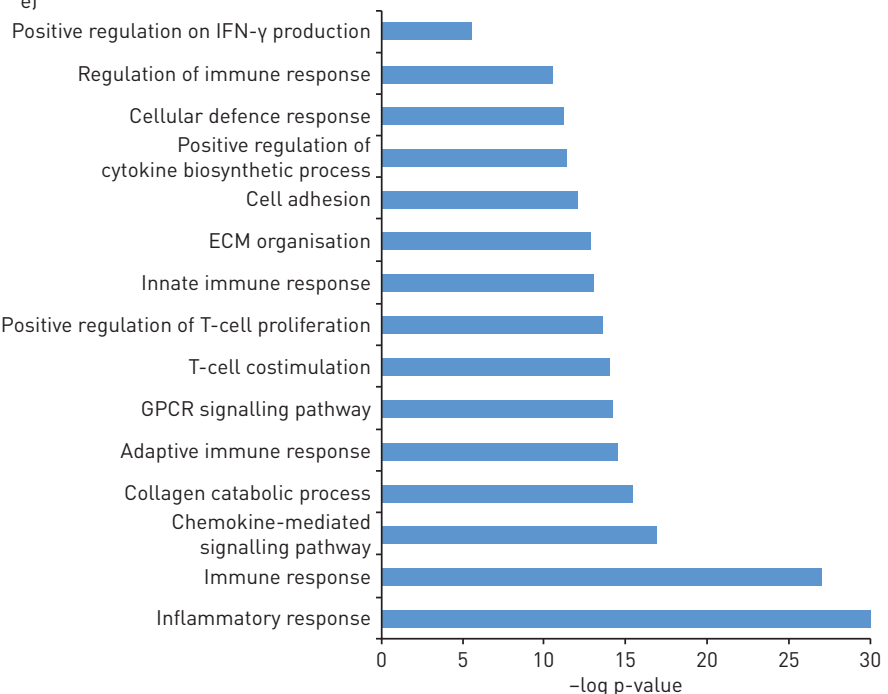

FIGURE 2 Principal component (PC)-informed analysis of the global gene expression patterns. CRSwNP-NP: nasal polyps tissues from patients with chronic rhinosinusitis with nasal polyps; CRSwNP-IT: paired inferior turbinate tissues from patients with chronic rhinosinusitis with nasal polyps; CS-IT: inferior turbinate in control subjects; PLC: phospholipase C; GPCR: G-protein coupled receptor; IFN: interferon; ECM: extracellular matrix. a) PC plot with sample clustering using the variance of axis 1 (PC1) and 2 (PC2). Box plots of b) PC1 and c) PC2 stratified by tissues: CRSwNP-NP, CRSwNP-IT and CS-IT. Gene Ontology of the biological processes associated with the top loading genes from d) PC1 and e) PC2.

(supplementary figure E1). This analysis validated the PCA findings, despite that CRSwNP-IT shared certain gene expression patterns with CRSwNP-NP.

Collectively, these findings highlight a more pronounced difference in gene signatures (particularly cilia-associated and inflammatory genes) in CRSwNP patients independent of the tissue type. This is critical since the inflammation in CRSwNP-IT could also contribute to disease.

\section{Pairwise comparisons identify more DEGs in NP than in control IT tissue}

Having delineated the global gene expression patterns among each tissue type, we next evaluated the DEGs for pairwise comparison among the tissues. For this purpose, we performed edgeR analysis on the quality controlled sequencing data using transcript-level counts. We first sought to evaluate the differences among nonpolyp IT tissues. Here, we only identified 915 DEGs for the CRSwNP-IT and CS-IT comparison that survived multiple testing (supplementary table E3), which corroborated our expectations from the PC-informed analysis. The top GO sets enriched by these DEGs were involved in the interferon (IFN) signalling pathway and viral responses (table 1 and supplementary table E4). By contrast, we identified a notably larger number of DEGs $(n=6182)$ when comparing CRSwNP-NP with CS-IT. These DEGs were involved in collagen processing and organisation, inflammatory responses, and $O$-glycan processing (table 1 and supplementary table E4). We then made a comparison between tissues from CRSwNP patients only, 
TABLE 1 Gene Ontology (GO) analysis for the biological processes associated with differentially expressed genes for pairwise analysis of nasal epithelial tissues

\begin{tabular}{|c|c|c|c|c|c|c|c|}
\hline GO identifier & Description & Annotated $\mathbf{n}$ & Significant $\mathrm{n}$ & Expected $n$ & p-value & Ontology & $\begin{array}{c}\text { Adjusted } \\
\text { p-value }\end{array}$ \\
\hline \multicolumn{8}{|c|}{ CRSwNP-IT vs CS-IT } \\
\hline G0:0060337 & Type I IFN signalling pathway & 49 & 21 & 2.85 & $4.00 \times 10^{-12}$ & $\mathrm{BP}$ & $2.89 \times 10^{-8}$ \\
\hline GO:0051607 & Defence response to virus & 155 & 36 & 9.01 & $9.90 \times 10^{-11}$ & $\mathrm{BP}$ & $3.57 \times 10^{-7}$ \\
\hline G0:0045071 & $\begin{array}{l}\text { Negative regulation of viral genome } \\
\text { replication }\end{array}$ & 39 & 13 & 2.27 & $1.50 \times 10^{-7}$ & $\mathrm{BP}$ & $3.61 \times 10^{-4}$ \\
\hline \multicolumn{8}{|c|}{ CRSwNP-NP vs CS-IT } \\
\hline G0:0030574 & Collagen catabolic process & 57 & 42 & 19.77 & $2.00 \times 10^{-9}$ & $\mathrm{BP}$ & $7.22 \times 10^{-6}$ \\
\hline G0:0016266 & O-glycan processing & 52 & 35 & 18.04 & $1.60 \times 10^{-6}$ & $\mathrm{BP}$ & $1.93 \times 10^{-3}$ \\
\hline G0:0006954 & Inflammatory response & 539 & 252 & 186.98 & $3.50 \times 10^{-6}$ & $\mathrm{BP}$ & $2.89 \times 10^{-3}$ \\
\hline G0:0030199 & Collagen fibril organisation & 35 & 25 & 12.14 & $1.00 \times 10^{-5}$ & $\mathrm{BP}$ & $6.56 \times 10^{-3}$ \\
\hline \multicolumn{8}{|l|}{ CRSwNP-NP vs } \\
\hline \multicolumn{8}{|l|}{ CRSWNP-IT } \\
\hline GO:0007155 & Cell adhesion & 1357 & 194 & 135.64 & $1.20 \times 10^{-9}$ & $\mathrm{BP}$ & $2.71 \times 10^{-6}$ \\
\hline GO:0030574 & Collagen catabolic process & 57 & 23 & 5.70 & $1.50 \times 10^{-9}$ & $\mathrm{BP}$ & $2.71 \times 10^{-6}$ \\
\hline G0:0060337 & Type I IFN signalling pathway & 49 & 18 & 4.90 & $1.50 \times 10^{-7}$ & $\mathrm{BP}$ & $2.17 \times 10^{-4}$ \\
\hline GO:0030199 & Collagen fibril organisation & 35 & 15 & 3.50 & $4.30 \times 10^{-7}$ & $\mathrm{BP}$ & $4.15 \times 10^{-4}$ \\
\hline G0:0001503 & Ossification & 286 & 61 & 28.59 & $6.50 \times 10^{-7}$ & $\mathrm{BP}$ & $5.21 \times 10^{-4}$ \\
\hline G0:0030593 & Neutrophil chemotaxis & 75 & 22 & 7.50 & $3.40 \times 10^{-6}$ & $\mathrm{BP}$ & $2.23 \times 10^{-3}$ \\
\hline GO:0007229 & Integrin-mediated signalling pathway & 80 & 22 & 8.00 & $7.50 \times 10^{-6}$ & $\mathrm{BP}$ & $3.92 \times 10^{-3}$ \\
\hline GO:0030198 & ECM organisation & 288 & 75 & 28.79 & $8.80 \times 10^{-6}$ & $\mathrm{BP}$ & $4.24 \times 10^{-3}$ \\
\hline G0:0001525 & Angiogenesis & 353 & 67 & 35.29 & $1.10 \times 10^{-5}$ & $\mathrm{BP}$ & $4.96 \times 10^{-3}$ \\
\hline GO:0022617 & ECM disassembly & 74 & 22 & 7.40 & $1.70 \times 10^{-5}$ & $\mathrm{BP}$ & $7.22 \times 10^{-3}$ \\
\hline GO:0006954 & Inflammatory response & 539 & 96 & 53.88 & $2.10 \times 10^{-5}$ & $\mathrm{BP}$ & $8.42 \times 10^{-3}$ \\
\hline
\end{tabular}

CRSwNP-IT: paired inferior turbinate tissues from patients with chronic rhinosinusitis with nasal polyps; CS-IT: inferior turbinate in control subjects; CRSwNP-NP: nasal polyps from patients with chronic rhinosinusitis with nasal polyps; IFN: interferon; ECM: extracellular matrix; BP: biological process.

which revealed 1592 DEGs. Interestingly, the GO analysis identified a broader set of significantly enriched pathways from these DEGs (table 1 and supplementary table E4) that ranged from collagen organisation, angiogenesis, ECM disassembly to inflammatory responses.

We next thoroughly examined the top DEGs (based on log fold change) for the pairwise comparisons (figure $3 \mathrm{a}-\mathrm{c}$ ). Interestingly, the DEGs in this analysis identified biological processes that were also identified in the previous analyses, such as collagen organisation and inflammatory responses (particularly IFN signalling). We additionally observed other significantly enriched biological processes, such as ECM disassembly, angiogenesis, ossification and neutrophil chemotaxis, that are reportedly involved in CRSwNP pathogenesis. Furthermore, we identified certain tissue-specific gene signatures such as CCL18 levels that were enriched in CRSwNP-NP compared with CRSwNP-IT and CS-IT (log fold change 6.48 and 6.85, respectively; both false discovery rate $<0.05$ ) (figure $3 \mathrm{~d}-\mathrm{f}$ ). The top DEGs for pairwise comparisons are detailed in supplementary tables E5-E7 and all DEGs for pairwise comparisons are listed in supplementary table E8.

Surprisingly, further analysis including the covariates indicative of the inflammatory phenotypes (eosinophilia and neutrophilia) did not reveal strong DEG signatures (supplementary table E9). After adjusting for multiple testing, only 16 and two DEGs were detected for eosinophilic and neutrophilic phenotypes, respectively. Analysis accounting for coexisting allergic rhinitis revealed only 35 DEGs, highlighting that allergic rhinitis was not a major confounder for our transcriptomic analysis. Moreover, atopic status conferred limited impact on the gene expression in CRSwNP patients and control subjects (supplementary table E10). However, caution should be exercised when interpreting this covariate analysis given the limited samples size for the stratified samples.

Finally, we compared the DEGs in each pairwise analysis and those that were shared among CRSwNP-IT, CRSwNP-NP and CS-IT (figure 4). The most unique DEGs were observed in the CRSwNP-NP versus CS-IT analysis; here, 4114 (66.54\%) DEGs were not detected in other analyses. Only 124 DEGs were shared among the three pairwise analyses and, as expected, the lowest count of unique DEGs (75 (8.19\%)) was found between CRSwNP-IT with CS-IT, suggesting strong differential expression in nonpolyp tissues. 
a)

d)
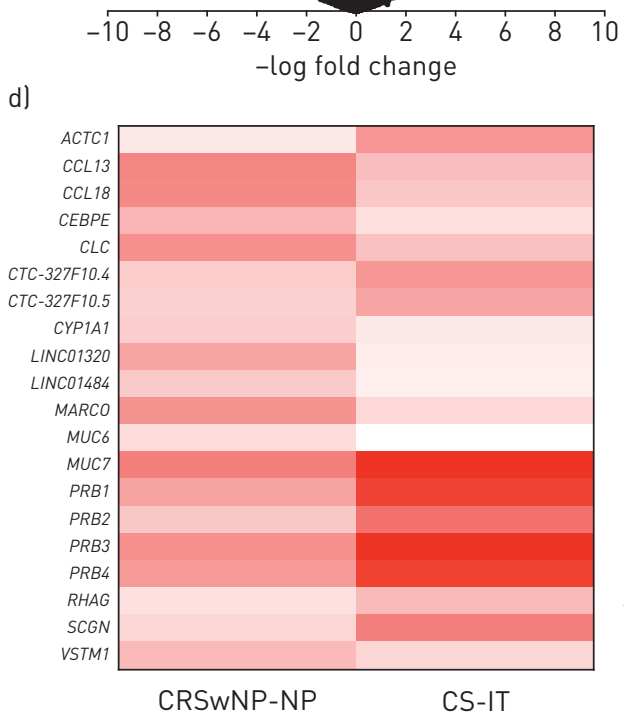

b)

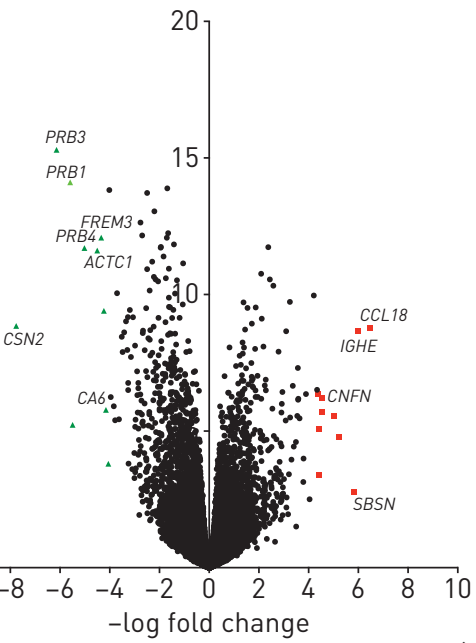

e)

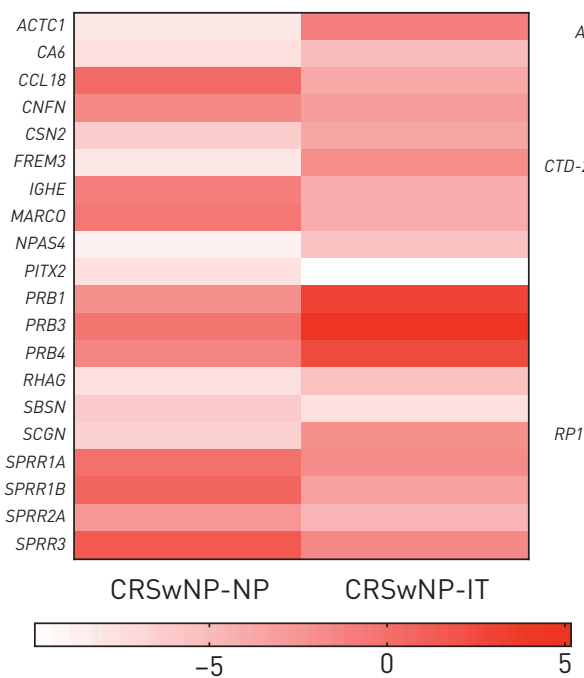

c)
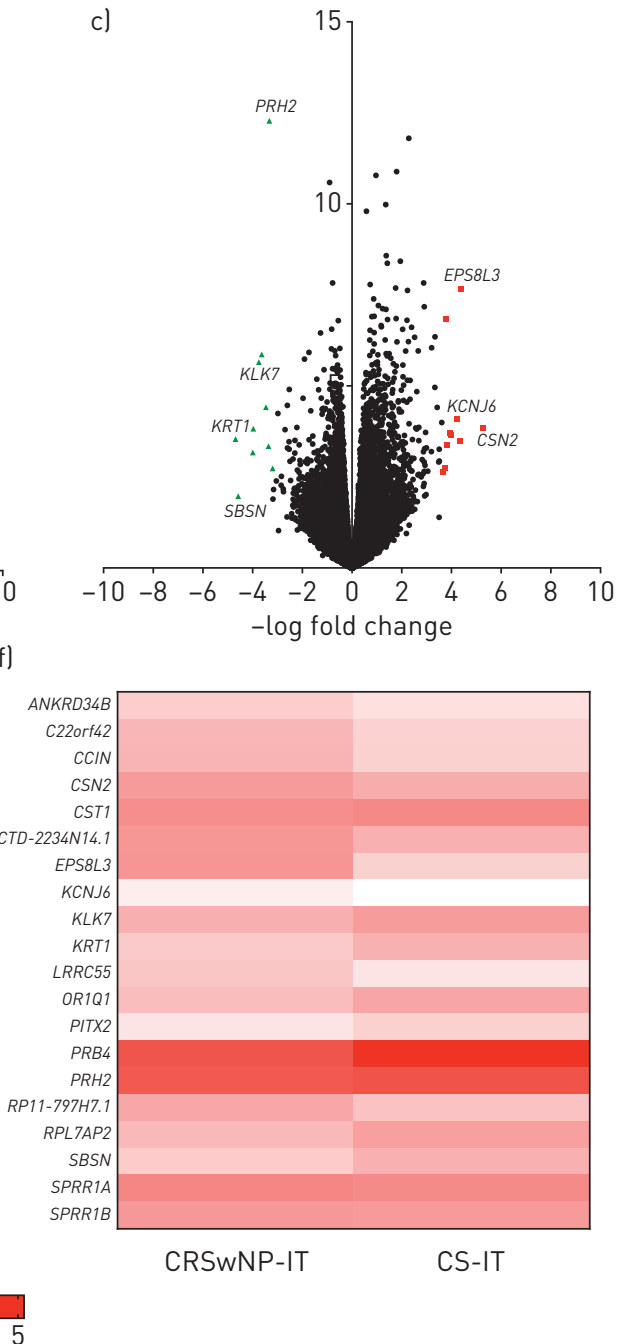

FIGURE 3 Differentially expressed genes (DEGs) stratified by tissue type. CRSwNP-NP: nasal polyps from patients with chronic rhinosinusitis with nasal polyps; CS-IT: inferior turbinate in control subjects; CRSWNP-IT: paired inferior turbinate tissues from patients with chronic rhinosinusitis with nasal polyps. Volcano plots showing the DEGs for a) CRSwNP-NP versus CS-IT, b) CRSwNP-NP versus CRSwNP-IT and c) CRSwNP-IT versus CS-IT. Positive log fold changes are shown in red and negative log fold changes are shown in green. $d-f$ ) Heat maps of the top 10 positive log fold changes and the top 10 negative log fold changes for each of the above comparisons.

Taken together, these findings revealed strong DEG signals related to inflammation, viral responses and airway remodelling in CRSwNP that were not confounded by the inflammatory phenotypes.

\section{IPA highlights axonal guidance signalling as the common pathway in CRSwNP-NP, CRSwNP-IT and CS-IT}

To determine the relevance of the identified DEGs to CRSwNP, we performed IPA on two sets of genes. For the first set, we used the 1387 DEGs shared $(124+1263)$ between the CRSwNP-NP versus CS-IT comparison and the CRSwNP-NP versus CRSwNP-IT comparison to identify enriched pathways. The top canonical pathways (determined by the percentage overlap for between-group comparisons) enriched for these sets of DEGs consisted of agranulocyte adhesion and diapedesis, axonal guidance and interleukin (IL)-8 signalling, and phagosome formation (table 2). For the second set, we analysed the $4795(681+4114)$ DEGs detected in the CRSwNP-NP versus CS-IT comparison, but not the CRSwNP-NP versus CRSwNP-IT comparison (table 2). These genes represent canonical pathways including molecular mechanisms of cancer, $\mathrm{N}$-formylmethionyl-leucyl-phenylalanine signalling in neutrophils, and phosphate and tension homology deleted on chromosome 10 and eukaryotic initiation factor-2 signalling. Axonal guidance signalling was a common canonical pathway to both analyses.

These findings imply that exaggerated inflammatory responses and epithelial growth may predispose NP formation and that axonal guidance signalling represents a critical target for NP management. 
FIGURE 4 Venn diagram representing the overlap between the differentially expressed genes (DEGs) among the three pairwise comparisons: paired inferior turbinate tissues from patients with chronic rhinosinusitis with nasal polyps (CRSwNP-IT) versus inferior turbinate in control subjects (CS-IT), nasal polyps from patients with chronic rhinosinusitis with nasal polyps (CRSwNP-NP) versus CRSWNP-IT and CRSWNP-NP versus CS-IT.

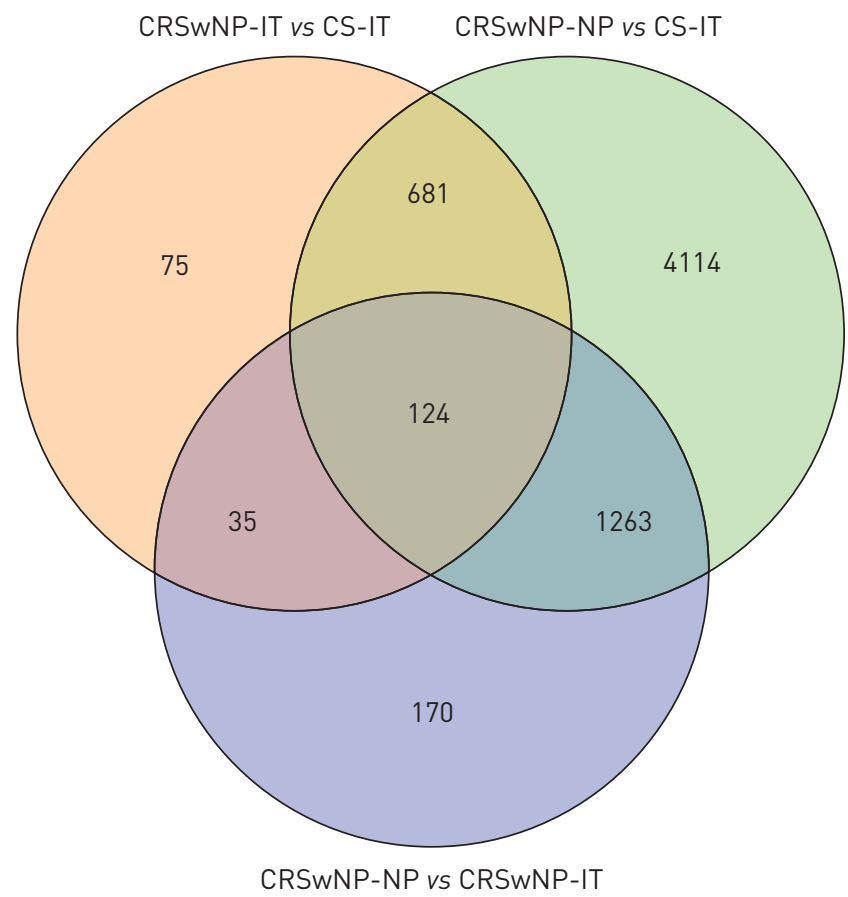

Enrichment analysis revealed gene sets associated with the inflammatory response and aberrant cell differentiation potentially involved in NP formation

Finally, we performed an enrichment analysis using GSEA to identify concordant gene expression patterns in CRSwNP-NP compared with CRSwNP-IT and CS-IT. Representative candidate enriched gene sets for CRSwNP-NP versus CS-IT revealed myosin filament, microtubule movement, and other epithelium integrity and function categories (table 3). In addition, we detected strong inflammatory response genes and those involved in inflammasome-associated IL-1 secretion. Interestingly, immune responses, such as immunological memory and monocyte or macrophage immune responses, were highly enriched in the CRSwNP-NP versus CRSwNP-IT comparisons, whereas the candidate gene sets of IFN-associated inflammation and keratinisation of epithelial cells were enriched in the CRSwNP-IT versus CS-IT comparisons (supplementary tables E11 and E12). We observed coherent and consistent gene sets involved

TABLE 2 The top canonical pathways for the differentially expressed gene (DEGs) identified between nasal polyps from patients with chronic rhinosinusitis with nasal polyps (CRSwNP-NP) versus inferior turbinate in control subjects (CS-IT) and CRSWNP-NP versus paired inferior turbinate tissues from patients with chronic rhinosinusitis with nasal polyps (CRSWNP-IT)

\begin{tabular}{lcc} 
Canonical pathway & p-value & Overlap n/N (\%) \\
\hline DEGs shared between CRSwNP-NP vs CS-IT and CRSwNP-NP vs CRSwNP-IT & \\
Agranulocyte adhesion and diapedesis & $1.23 \times 10^{-10}$ & $35 / 193(18.1)$ \\
Axonal guidance signalling & $4.99 \times 10^{-10}$ & $58 / 457(12.7)$ \\
Phagosome formation & $8.58 \times 10^{-10}$ & $27 / 131(20.6)$ \\
Interleukin-8 signalling & $2.65 \times 10^{-8}$ & $32 / 203(15.8)$ \\
Hepatic stellate cell activation & $4.78 \times 10^{-8}$ & $30 / 187(16.0)$ \\
DEGs unique for CRSwNP-NP vs CS-IT and not identified in CRSwNP-NP vs CRSwNP-IT & \\
Molecular mechanisms of cancer & $5.62 \times 10^{-9}$ & $107 / 394(27.2)$ \\
fMLP signalling in neutrophils & $2.87 \times 10^{-8}$ & $46 / 129(35.7)$ \\
Axonal guidance signalling & $4.18 \times 10^{-8}$ & $117 / 457(25.6)$ \\
PTEN signalling & $8.79 \times 10^{-8}$ & $44 / 125(35.2)$ \\
EIF-2 signalling & $1.42 \times 10^{-7}$ & $67 / 227(29.5)$
\end{tabular}

fMLP: N-formylmethionyl-leucyl-phenylalanine; PTEN: phosphate and tension homology deleted on chromosome 10; EIF-2: eukaryotic initiation factor-2. 
TABLE 3 Candidate enriched gene sets associated with the comparison between nasal polyps from patients with chronic rhinosinusitis with nasal polyps (CRSwNP-NP) and paired inferior turbinate tissues from patients with chronic rhinosinusitis with nasal polyps (CRSWNP-IT)

\begin{tabular}{|c|c|c|c|c|}
\hline Name & $\begin{array}{l}\text { GSEA } \\
\text { identifier }\end{array}$ & p-value & NES & Leading edge \\
\hline Myosin filament & M17213 & $2.40 \times 10^{-3}$ & -2.01 & ACTG2, MYH11, MYBPC1, MYOM1 \\
\hline 0 -glycan biosynthesis & M6929 & $7.21 \times 10^{-3}$ & -1.72 & $\begin{array}{c}\text { GALNT3, C1GALT1, GALNT1, ST3GAL1, GALNT4, GCNT3, GALNT6, GALNT13, } \\
\text { B3GNT6, GALNT17, GALNT15, GCNT4, GCNT4 }\end{array}$ \\
\hline ErbB network pathway & M201 & $2.15 \times 10^{-2}$ & -1.64 & $E G F, E R B B 4, B T C, N R G 3, N R G 4$ \\
\hline $\begin{array}{l}\text { Milicic familial adenomatous } \\
\text { polyposis }\end{array}$ & M9371 & $4.66 \times 10^{-2}$ & 1.50 & LCN2, SLCO1B3, CDH3, XIST, CEMIP \\
\hline Interleukin-1 secretion & M2321 & $3.28 \times 10^{-2}$ & 1.53 & NLRP3, NLRP2, NLRC4, NLRP12, ABCA1, PYCARD \\
\hline Inflammatory response & M13063 & $5.17 \times 10^{-3}$ & 1.76 & IDO1, SYK, IL4R, ALOX5, LYN, P2RX1, FCER1G \\
\hline Cancer module 211 & M15032 & $1.74 \times 10^{-3}$ & 2.04 & MMP9, MMP2, MMP1, MMP3, MMP7, MMP13, CHIT1, MMP10, MMP11 \\
\hline $\begin{array}{l}\text { Multiple myeloma } \\
\text { upregulated genes }\end{array}$ & M4888 & $1.59 \times 10^{-3}$ & 2.33 & $\begin{array}{c}\text { BIRC5, EZH2, AURKA, CDK1, TYMS, FOXM1, TOP2A, CCNB1, KPNA2, BUB1B, } \\
\text { TTK, UBE2C, NEK2, RRM2, TPX2, RACGAP1, ASPM, PCLAF, FANCI, KIF14, } \\
\text { NUF2, MCM10, CCNB2, UBE2T, DLGAP5, SKA1, NUSAP1, E2F8, PRR11, SPC25, } \\
\text { DEPDC1B }\end{array}$ \\
\hline
\end{tabular}

in the adaptive immune response that were upregulated in CRSwNP-NP versus CRSwNP-IT (figure 5a), including regulatory $\mathrm{T}$-cell (Treg) molecules such as FOXP3, complement genes such as C4BPA and $C 4 B P B$, ILs such as IL4,IL4R and IL1RL1, and protein kinase PTPRC. Another interesting candidate pathway between CRSwNP-NP and CRSwNP-IT involved IFN signatures that were usually observed in cancer (figure 5b). Importantly, genes such as STAT1, IFIT1, IFIT3 and IRF9 were all consistently downregulated in CRSwNP-NP compared with paired IT tissue (CRSwNP-IT), as observed in many cancer tissues. These findings indicate that the inflammation in CRSwNP-NP due to infections might be mediated by IFN-stimulated genes.

We found a more generic inflammatory response network identified through GSEA when comparing CRSwNP-NP with CS-IT (figure 5c). However, this inflammatory response could be associated with CRS pathogenesis, NP formation or both. Lastly, when comparing CRSwNP-IT and CS-IT, we found evidence of persistent inflammation mediated through a lack of tolerance induction (figure 5d). Interestingly, key genes involved in immunoregulation and Treg function included FOXP3, IL2RA, IDO1, IRAK3 and HLAG, suggesting strong inflammation in the nonpolyp tissues in patients with CRSwNP, and the lack of tolerance might potentially lead to NP formation. Details of all three comparisons are provided in supplementary table E13.

Taken together, these data indicate that gene sets associated with the inflammatory and immune responses are enriched in NP, and support that inflammation and aberrant cell differentiation underlie early-stage NP formation.

\section{Discussion}

We performed whole-transcriptomic sequencing followed by pairwise comparisons of CRSwNP-NP, CRSwNP-IT and CS-IT, which allowed us to identify candidate genes and canonical pathways. The global gene expression patterns differed most dramatically between CRSwNP-NP and CS-IT, whereas CRSwNP-IT shared a certain degree of overlapping gene signatures with CS-IT. Apart from the identification of DEGs associated with ECM disassembly, O-glycan processing and host viral response, we revealed a novel association for NP to axonal guidance signalling as a common pathway and the potential induction of immune intolerance associated with persistent airway inflammation in NP.

CRS pathogenesis with NP formation follows sequential events, including environmental triggers (i.e. allergen sensitisation, Staphylococcus aureus infections), heightened inflammation, angiogenesis and tissue remodelling $[1,27]$. Intact epithelial barrier function is essential to protect the airways from allergens or pathogenic insults. Indeed, our PC-informed analysis revealed genes associated with ciliary assembly and function and 
a)

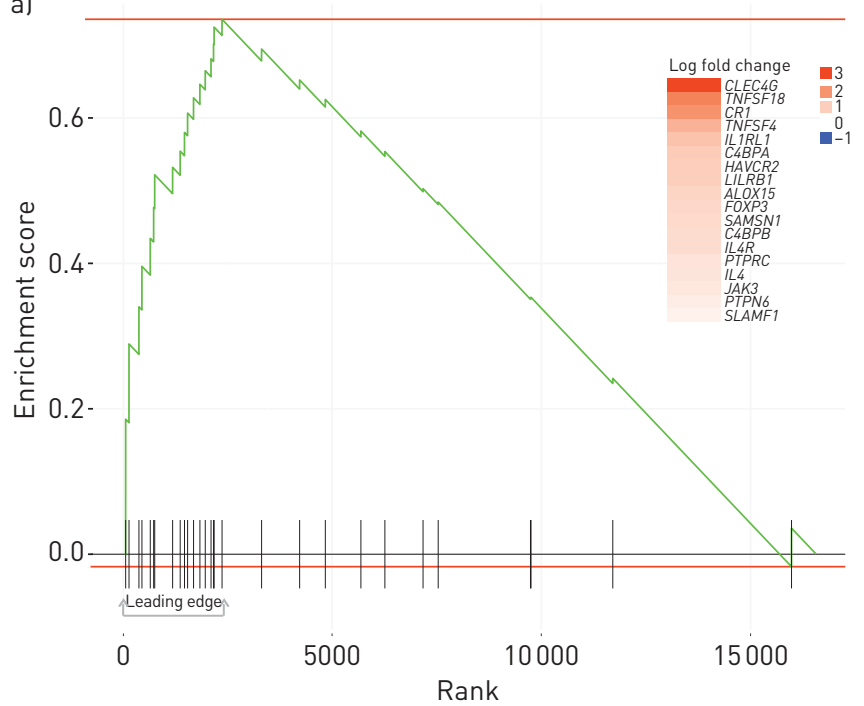

c) 0.8

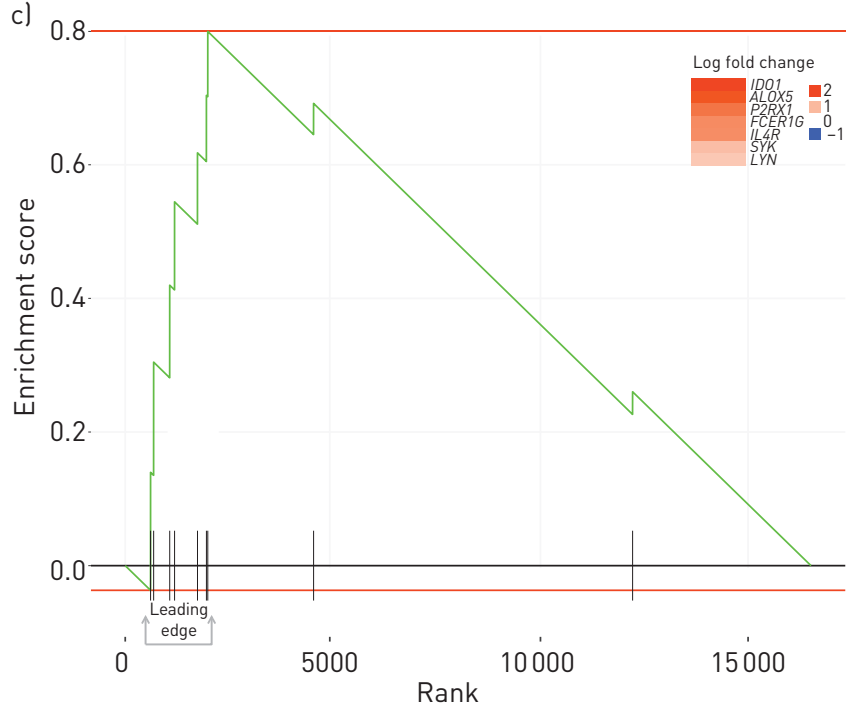

b)

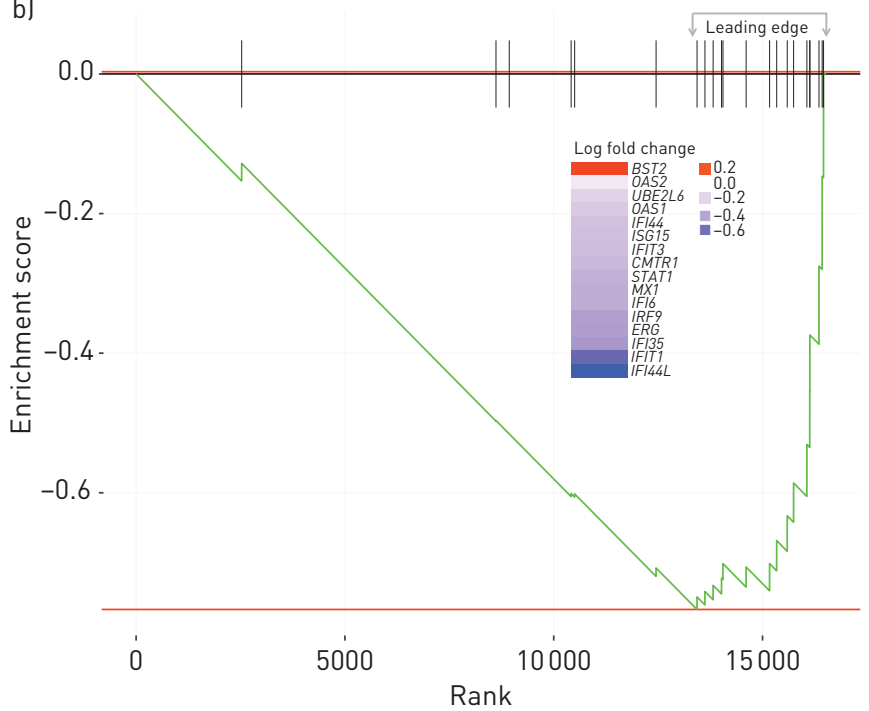

d)

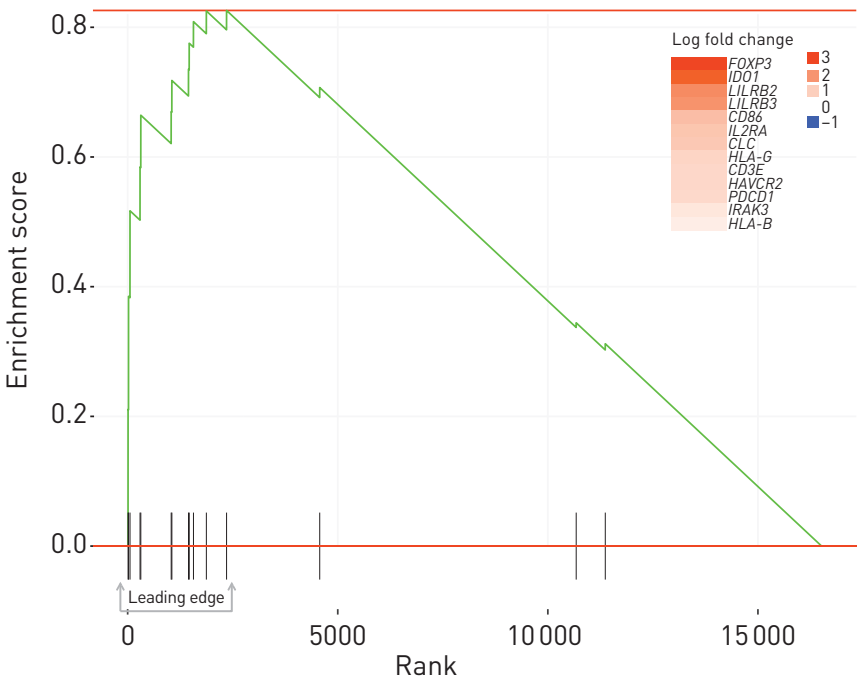

FIGURE 5 Enrichment plots of key pathways identified from the gene set analysis. CRSwNP-NP: nasal polyps from patients with chronic rhinosinusitis with nasal polyps; CRSwNP-IT: paired inferior turbinate tissues from patients with chronic rhinosinusitis with nasal polyps; CS-IT: inferior turbinate in control subjects. a, b) Selected enrichment plots for the pairwise CRSwNP-NP versus CRSwNP-IT comparisons: al negative regulation of adaptive immune response and b) Einav interferon signature in cancer. Selected pathways for the C) CRSwNP-NP versus CS-IT and d) CRSwNP-NP versus CS-IT comparisons: c) molecular mediators in inflammatory response and d) regulation of tolerance induction.

immune responses, which echoed previous studies regarding aberrant ciliary ultrastructural marker expression, mislocalisation of ciliogenesis markers and aberrant immune function in CRSwNP $[28,29]$. Importantly, our GO analysis highlighted the interactions between inflammation (and possibly chronic latent viral infection), defective host defences (cellular immunity and impaired mucociliary clearance) and airway remodelling in driving CRSwNP. Because these signatures could have stemmed from the interaction between impaired mucociliary clearance and tissue-specific inflammation, we made further comparisons between CRSwNP-NP and CRSwNP-IT [2]. These comparisons revealed DEGs involved in collagen and ECM metabolism, inflammatory responses, ossification, and angiogenesis, reaffirming the roles of a heightened IFN signalling response [30], angiogenesis [31], ossification [32], increased matrix metalloproteinase expression [33], and collagen synthesis and deposition [34] in modulating CRSwNP formation.

Pathway analyses revealed canonical pathways that mediate NP formation. The DEGs shared between CRSwNP-NP versus CS-IT and CRSwNP-NP versus CRSwNP-IT comparisons revealed the axonal guidance signalling pathway [35], which reportedly correlates with chronic airway inflammation [36]. Interestingly, the unique DEGs for the aforementioned comparisons revealed that pathways associated with the molecular mechanisms of cancer and neutrophil $N$-formylmethionyl-leucyl-phenylalanine signalling drive polyp formation. Suppressing exaggerated cell growth might, therefore, be an effective management 
approach for CRSwNP in the future, given that the rapid recurrence of CRSwNP mimics benign tumour growth.

We further explored the gene sets enriched among the the various nasal tissues. Gene sets associated with epithelial differentiation and IFN responses were implicated in CRSwNP-NP versus CS-IT, which partially echoed our findings by IPA. Importantly, comparing CRSwNP-NP and CRSwNP-IT revealed the gene sets associated with inflammatory (particularly IL-1) responses, O-glycan biosynthesis and NP formation, which are likely the crucial risk factors that modulate early-stage CRSwNP. Significantly altered IFN-stimulated gene expression implicates the biological significance of abnormal antiviral responses in inducing NP formation.

Although eosinophilia reportedly predisposes NP formation, our analysis based on eosinophil enumeration surprisingly revealed finite DEGs $(\mathrm{n}=35$ at false discovery rate $<0.05)$, suggesting that eosinophilia was not a major confounder. Despite a previous study identifying the top DEGs such as CST1 and CCL18 in eosinophilic NP, no three-way pairwise comparisons such as ours have been made to control for false positives [37]. Similarly, coexisting allergic rhinitis did not yield strong DEGs.

Our study has important clinical implications. Our analytical pipeline has robustly identified the gene signatures and their associated signalling pathways and gene sets based on three pairwise comparisons with CS-IT as nondisease controls and CRSwNP-IT as disease controls. This approach minimised false-positive findings arising from the interactions between environmental and genetic factors. These DEGs represent a valuable database for future functional validation of gene expression patterns (i.e. cilia and mucin genes) in vivo and in vitro. The immune intolerance has shed light on a novel target for the management of CRSwNP, indicating the potential roles of immunomodulators. Furthermore, our findings may guide the selection of gene signatures to determine the efficacy of therapeutic interventions (including biologics such as mepolizumab, benralizumab and omalizumab) for future clinical trials.

Some limitations should be addressed. First, we sequenced heterogeneous tissues with multiple cell types and cell deconvolution was not conducted. Future studies that account for various cell types are needed. Second, we did not repeatedly sample the tissues, which might help address the differential gene expression before and after surgery or medication therapy (i.e. biologic exposure), nor did we identify the cardinal culprit DEGs responsible for recurrence of CRSwNP. Third, we only evaluated upper airway inflammation, but not systemic inflammation (i.e. eosinophilic versus noneosinophilic) via blood sampling. Fourth, the IT is not an ideal anatomical site to compare gene expression patterns with NP; however, the middle turbinate is frequently occupied by large-sized NP (NP size score $>1$ ), which renders surgical sampling challenging. Nonetheless, we found strong evidence of similar inflammatory responses in CRSwNP-NP and CRSwNP-IT, and the airway epithelium of CRSwNP-NP was similar overall to CRSwNP-IT. The prominent gene signatures associated with inflammatory responses in CRSwNP-IT indicate that the IT (control group) remains a valid source for sequencing investigations. Finally, we did not adjust RNA expression levels with age because it explained only a minority of the total variation.

In summary, we performed whole-transcriptomic sequencing with a novel three-way pairwise comparison, and identified in the CRSwNP-IT versus CS-IT comparison the important roles of gene sets orchestrating immune intolerance and a dysregulated immune response (i.e. defective Treg response), which may provide targets for therapeutic interventions. Our study provides valuable information for future investigations to functionally validate the gene expression patterns of defective host defences (including cilia dysfunction and immune dysregulation), inflammation and abnormal metabolism of the ECM, and to explore the biological responses to therapeutic interventions in CRSwNP.

Acknowledgements: The authors would like to thank the study participants who donated their samples for the advancement of scientific knowledge. The authors would also like to thank Jessica Tamanini from Insight Editing London (London, UK) for editing assistance for the manuscript.

Author contributions: Conceived and designed the experiments: D.Y. Wang, A.K. Andiappan and W-J. Guan. Collection of samples: Y. Peng, X-X. Zi, T-F. Tian, L. Shi, J. Ye and Q-H. Qiu. Performed the experiments: Y. Peng, X-X. Zi, T-F. Tian and A.K. Andiappan. Data analysis: A.K. Andiappan, Y. Peng, X-X. Zi, T-F. Tian, W-J. Guan, B. Lee, J. Lum, S.A. Tang and K.S. Tan. Contributed reagents/materials/analysis tools: A.K. Andiappan and D.Y. Wang. Wrote the paper: W-J. Guan, A.K. Andiappan, K.S. Tan, Y. Peng, X-X. Zi, T-F. Tian. Critical review and approval: A.K. Andiappan, D.Y. Wang and W-J. Guan.

Conflict of interest: Y. Peng has nothing to disclose. X-X. Zi has nothing to disclose. T-F. Tian has nothing to disclose. B. Lee has nothing to disclose. J. Lum has nothing to disclose. S.A. Tang has nothing to disclose. K.S. Tan has nothing to disclose. Q-H. Qiu has nothing to disclose. J. Ye has nothing to disclose. L. Shi reports grants from The Key Research Development Program of Shandong Province (2018CXGC1214), outside the submitted work. W-J. Guan reports grants from Guangdong Province Universities and Colleges Pearl River Scholar Funded Scheme 2017, during the conduct of the study. A.K. Andiappan reports grants from National Medical Research Council of Singapore (OFYIRG17nov065), 
during the conduct of the study. D.Y. Wang reports grants from National Medical Research Council of Singapore (NMRC/CIRG/1458/2016), outside the submitted work.

Support statement: Supported by National Medical Research Council of Singapore (NMRC/CIRG/1458/2016; D.Y. Wang), National Medical Research Council of Singapore (OFYIRG17nov065; A.K. Andiappan), The Key Research Development Program of Shandong Province (2018CXGC1214; L. Shi), Guangdong Province Universities and Colleges Pearl River Scholar Funded Scheme 2017 (201710010097; W-J. Guan), and the National Nature Science Foundation of China (81873690; Q-H. Qiu). Funding information for this article has been deposited with the Crossref Funder Registry.

\section{References}

$1 \quad$ Fokkens WJ, Lund VJ, Mullol J, et al. EPOS 2012: European position paper on rhinosinusitis and nasal polyps 2012. A summary for otorhinolaryngologists. Rhinology 2012; 50: 1-12.

2 Stevens WW, Schleimer RP, Kern RC. Chronic rhinosinusitis with nasal polyps. J Allergy Clin Immunol Pract 2016; 4: 565-572.

3 Zhang Y, Gevaert E, Lou H, et al. Chronic rhinosinusitis in Asia. J Allergy Clin Immunol 2017; 140: 1230-1239.

4 Bachert C, Zhang L, Gevaert P. Current and future treatment options for adult chronic rhinosinusitis: focus on nasal polyposis. J Allergy Clin Immunol 2015; 136: 1431-1440.

5 Richer SL, Truong-Tran AQ, Conley DB, et al. Epithelial genes in chronic rhinosinusitis with and without nasal polyps. Am J Rhinol 2008; 22: 228-234.

6 Jiao J, Wang M, Duan S, et al. Transforming growth factor-betal decreases epithelial tight junction integrity in chronic rhinosinusitis with nasal polyps. J Allergy Clin Immunol 2018; 141: 1160-1163.

7 Lima JT, Paula FE, Proenca-Modena JL, et al. The seasonality of respiratory viruses in patients with chronic rhinosinusitis. Am J Rhinol Allergy 2015; 29: 19-22.

8 Picelli S, Faridani OR, Bjorklund AK, et al. Full-length RNA-seq from single cells using Smart-seq2. Nat Protocol 2014; 9: 171-181.

9 Ding GQ, Zheng CQ, Bagga SS. Up-regulation of the mucosal epidermal growth factor receptor gene in chronic rhinosinusitis and nasal polyposis. Arch Otolaryngol Head Neck Surg 2007; 133: 1097-1103.

10 Jiao J, Duan S, Meng N, et al. Role of IFN-gamma, IL-13, and IL-17 on mucociliary differentiation of nasal epithelial cells in chronic rhinosinusitis with nasal polyps. Clin Exp Allergy 2016; 46: 449-460.

11 Liu J, Li YY, Andiappan AK, et al. Role of IL-13Ralpha2 in modulating IL-13-induced MUC5AC and ciliary changes in healthy and CRSwNP mucosa. Allergy 2018; 73: 1673-1685.

12 Qiu Q, Peng Y, Zhu Z, et al. Absence or mislocalization of DNAH5 is a characteristic marker for motile ciliary abnormality in nasal polyps. Laryngoscope 2018; 128: E97-E104.

13 Ding GQ, Zheng CQ. The expression of MUC5AC and MUC5B mucin genes in the mucosa of chronic rhinosinusitis and nasal polyposis. Am J Rhinol 2007; 21: 359-366.

14 Wang W, Gao Z, Wang H, et al. Transcriptome analysis reveals distinct gene expression profiles in eosinophilic and noneosinophilic chronic rhinosinusitis with nasal polyps. Sci Rep 2016; 6: 26604.

15 Anand VK, Kacker A, Orjuela AF, et al. Inflammatory pathway gene expression in chronic rhinosinusitis. Am J Rhinol 2006; 20: 471-476.

16 Payne SC, Han JK, Huyett P, et al. Microarray analysis of distinct gene transcription profiles in non-eosinophilic chronic sinusitis with nasal polyps. Am J Rhinol 2008; 22: 568-581.

17 Liu Z, Kim J, Sypek JP, et al. Gene expression profiles in human nasal polyp tissues studied by means of DNA microarray. J Allergy Clin Immunol 2004; 114: 783-790.

18 Benson M, Carlsson L, Adner M, et al. Gene profiling reveals increased expression of uteroglobin and other anti-inflammatory genes in glucocorticoid-treated nasal polyps. J Allergy Clin Immunol 2004; 113: 1137-1143.

19 Ordovas-Montanes J, Dwyer DF, Nyquist SK, et al. Allergic inflammatory memory in human respiratory epithelial progenitor cells. Nature 2018; 560: 649-654.

20 Tokunaga T, Ninomiya T, Kato Y, et al. The significant expression of TRPV3 in nasal polyps of eosinophilic chronic rhinosinusitis. Allergol Int 2017; 66: 610-616.

21 Yick CY, Zwinderman AH, Kunst PW, et al. Gene expression profiling of laser microdissected airway smooth muscle tissue in asthma and atopy. Allergy 2014; 69: 1233-1240.

22 Yick CY, Zwinderman AH, Kunst PW, et al. Transcriptome sequencing (RNA-Seq) of human endobronchial biopsies: asthma versus controls. Eur Respir J 2013; 42: 662-670.

23 Milara J, Morell A, Ballester B, et al. MUC4 impairs the anti-inflammatory effects of corticosteroids in patients with chronic rhinosinusitis with nasal polyps. J Allergy Clin Immunol 2017; 139: 855-862.

24 Milara J, Peiro T, Armengot M, et al. Mucin1 downregulation associates with corticosteroid resistance in chronic rhinosinusitis with nasal polyps. J Allergy Clin Immunol 2015; 135: 470-476.

25 Gao T, Ng CL, Li C, et al. Smoking is an independent association of squamous metaplasia in Chinese nasal polyps. Int Forum Allergy Rhinol 2016; 6: 66-74.

26 Cao PP, Li HB, Wang BF, et al. Distinct immunopathologic characteristics of various types of chronic rhinosinusitis in adult Chinese. J Allergy Clin Immunol 2009; 124: 478-484.

27 Valera FCP, Ruffin M, Adam D, et al. Staphylococcus aureus impairs sinonasal epithelial repair: effects in patients with chronic rhinosinusitis with nasal polyps and control subjects. J Allergy Clin Immunol 2019; 143: 591-603.

28 Peng Y, Guan WJ, Tan KS, et al. Aberrant localization of FOXJ1 correlates with the disease severity and comorbidities in patients with nasal polyps. Allergy Asthma Clin Immunol 2018; 14: 71.

29 Detwiller KY, Smith TL, Alt JA, et al. Differential expression of innate immunity genes in chronic rhinosinusitis. Am J Rhinol Allergy 2014; 28: 374-377.

30 Jang YJ, Lim JY, Kim S, et al. Enhanced interferon- $\beta$ response contributes to eosinophilic chronic rhinosinusitis. Front Immunol 2018; 9: 2330.

31 Shin JM, Byun JY, Baek BJ, et al. Cellular proliferation and angiogenesis in nasal polyps of young adult and geriatric patients. Int Forum Allergy Rhinol 2015; 5: 541-546.

32 Kim YK, Kim HJ, Kim J, et al. Nasal polyps with metaplastic ossification: CT and MR imaging findings. Neuroradiology 2010; 52: 1179-1184. 
33 Wang LF, Chien CY, Chiang FY, et al. Corelationship between matrix metalloproteinase 2 and 9 expression and severity of chronic rhinosinusitis with nasal polyposis. Am J Rhinol Allergy 2012; 26: e1-e4.

34 de Borja Callejas F, Martinez-Anton A, Picado C, et al. Corticosteroid treatment regulates mucosal remodeling in chronic rhinosinusitis with nasal polyps. Laryngoscope 2015; 125: E158-E167.

$35 \mathrm{Wu}$ D, Mueller SK, Nocera AL, et al. Axonal guidance signaling pathway is suppressed in human nasal polyps. Am J Rhinol Allergy 2018; 32: 208-216.

36 Okano M, Fujiwara T, Kariya S, et al. Regulatory effect of TLR3 signaling on staphylococcal enterotoxin-induced IL-5, IL-13, IL-17A and IFN-gamma production in chronic rhinosinusitis with nasal polyps. Allergol Int 2016; 65: 96-102.

37 Workman AD, Nocera AL, Mueller SK, et al. Translating transcription: proteomics in chronic rhinosinusitis with nasal polyps reveals significant discordance with messenger RNA expression. Int Forum Allergy Rhinol 2019; 9: 776-786. 